\title{
An Efficient MAC Protocol for Underwater Multi-user Uplink Communication Networks
}

\author{
Yu Luo ${ }^{\mathrm{a}}$, Lina $\mathrm{Pu}^{\mathrm{a}}$, Zheng Peng ${ }^{\mathrm{a}}$, Zhong Zhou ${ }^{\mathrm{b}}$, Jun-Hong Cui ${ }^{\mathrm{a}}$ \\ ${ }^{a}$ Computer Science \& Engineering Department, University of Connecticut, Storrs, CT \\ Email: \{yu.luo, lina.pu, zhengpeng, jcui\}@engr.uconn.edu \\ ${ }^{b}$ Amazon Inc., Seattle, USA
}

\begin{abstract}
Multi-user uplink communication has been proved to be a promising technique for spectrumefficient communications. However, due to the unique features of underwater acoustic networks (UANs), such as the long propagation delay, low transmission rate and long preamble of acoustic modems, conventional medium access control (MAC) protocols for terrestrial multi-user uplink communications need an overhaul to work efficiently in UANs. In this paper, we carefully consider these features and propose a new MAC protocol, called the competitive transmission MAC (CTMAC), for underwater multi-user uplink networks. In CT-MAC, we aim to improve the channel utilization and the energy efficiency of the protocol by using a parallel competition mechanism. With parallel competitions, the control packet produced by each user only need to reach the direct neighbors with a low transmission power to save energy. Meanwhile, the data generated by users in different time slots can join the competition transmission in parallel to improve the competition efficiency. In addition, we propose two competition schemes for CT-MAC to achieve both the short-term and the long-term fairness in different network conditions. The theoretical analysis and simulation results illustrate that, compared with the conventional multi-user uplink MAC protocols, CT-MAC can achieve higher channel utilization and much lower end-to-end delay in UANs, while maintaining comparable energy efficiency.
\end{abstract}

Keywords: Underwater Acoustic Networks (UANs), multi-user uplink communications, MAC protocol, competition transmission. 


\section{Introduction}

Underwater acoustic networks (UANs) have a wide range of applications in offshore structure flaw detection, underwater environment monitoring, target tracking and oceanography data collection $[1,2,3]$. The communication bandwidth of acoustic signal is very narrow (only tens of kilohertz) due to the heavy frequency-dependent attenuation [4]. Therefore, techniques like multiuser uplink communications, which can improve the spectrum efficiency, have attracted growing interests from both academia and industry.

In an underwater multi-user uplink network, a group of users communicate to a common receiver, such as a sink node or a surface buoy. By installing plural hydrophones, the receiver can decode multiple packets from different users simultaneously without any collision. In the past decades, there have been significant research efforts on the physical layer of multi-user uplink communications $[5,6,7]$. However, to make this technique practical in UANs, we need an efficient medium access control (MAC) protocol to coordinate the activities (sending and receiving) of each user for high channel utilization and energy efficiency purposes.

Extensive studies have been conducted on multi-user MAC protocol design for terrestrial uplink communication networks $[8,9]$. In channel aware Aloha [8], the receiver sends a feedback message after each successful data reception to provide the channel state information for senders. Only users with good channel qualities are allowed to send their data. By retarding the transmission of users with poor channel qualities, this scheme achieves good energy efficiency. In multi-antenna reception MAC [9], the sender and the receiver use two-way handshakes to reserve the channel. Once a receiver gets a sending request, it will broadcast a receive capability broadcast (RCBC) packet to inform the surrounding users the remaining number of senders it can support to send the data at the same time. Users stop sending requests if no more sender can be supported by the receiver.

Although the aforementioned MAC protocols work well in terrestrial multi-user uplink networks, they still need an overhaul to work efficiently in underwater networks, which have large propagation delays, low transmission rates and long preamble acoustic modems [10,11]. These features may introduce significant overhead in a network by increasing the collision probability, transmission time and energy consumption of control packets [11], or make the instantaneous channel state information (CSI) unavailable for dynamic resource (time, power and frequency) allocation [12]. 
However, the research on the MAC design for underwater multi-user uplink communications is rare. The authors in [13] designed a cross-layer protocol, UMIMO-MAC, for MIMO underwater networks. UMIMO-MAC utilize joint optimization on transmission power and transmission mode to improve the communication performance. However, the two-way handshake mechanism suffers the large propagation delay and long preamble problems in underwater multi-user uplink networks. This lead us to design a new protocol, which is called the competitive transmission MAC (CTMAC), to solve these problems.

In this paper, we do not consider the power allocation and the space-time coding issues, which have been well explored by researchers at the physical layer $[14,15,16]$, but focus on the problem that how to efficiently coordinate the activities of users in an underwater multi-user uplink network at the MAC layer. We aim to improve the network performance in terms of fairness, channel utilization and energy efficiency.

To achieve the above goals, in CT-MAC each user only uses a small transmission power to broadcast the control packet to its direct neighbors instead of the whole network. This is very important to reduce the energy consumption and the propagation delay on control packets. At the same time, a parallel competition mechanism is designed for CT-MAC to allow users with new data packets to quickly participate in a new round of competition before the old one is announced. In this way, a user is able to join in multiple rounds of competition in parallel, which significantly improves the competition efficiency of the protocol.

In order to have a fair transmission in different network conditions, we also propose two specific competition schemes for CT-MAC. The theoretical analysis and simulation results show that CT-MAC can achieve both the short-term and the long-term fairness in different network scenarios. Additionally, compared to the channel aware Aloha and the multi-antenna reception MAC, CT-MAC can achieve higher channel utilization and much lower end-to-end delay, while maintaining comparable energy efficiency. Especially in the high traffic generation rate scenario, the channel utilization of CT-MAC is about 3.15 and 3.4 times higher than the two representative MAC protocols, respectively.

The rest of the paper is organized as follows. We briefly describe the architecture of underwater uplink communication networks in Section 2. Then we discuss the motivation of our work in Section 3. In Section 4, we introduce CT-MAC in one-dimensional and two-dimensional multi-user 
uplink UANs. Two competition schemes for fair transmissions are proposed in Section 5. We brief the power allocation strategies supported by CT-MAC in Section6. Two special issues, namely, the bad request issue and the lost competition packet problem, are analyzed in Section 7 and Section 8 , respectively. The performance of CT-MAC is evaluated in Section 9. Finally, we conclude the paper in Section 10.

\section{Network Architecture}

In this paper, we consider the underwater uplink communication networks, in which a surface buoy or a sink node works as a common receiver to collect data from a group of underwater static users, as shown in Fig. 1. Such networks are usually used for the offshore instrument (e.g. wind farm and oil pipeline) health monitoring, underwater data collection and target detection [17]. We assume that each user uses the orthogonal frequency-division multiplexing (OFDM) modulation scheme, which has the ability to cope with severe frequency-selective fading due to multipath in underwater channel [18].

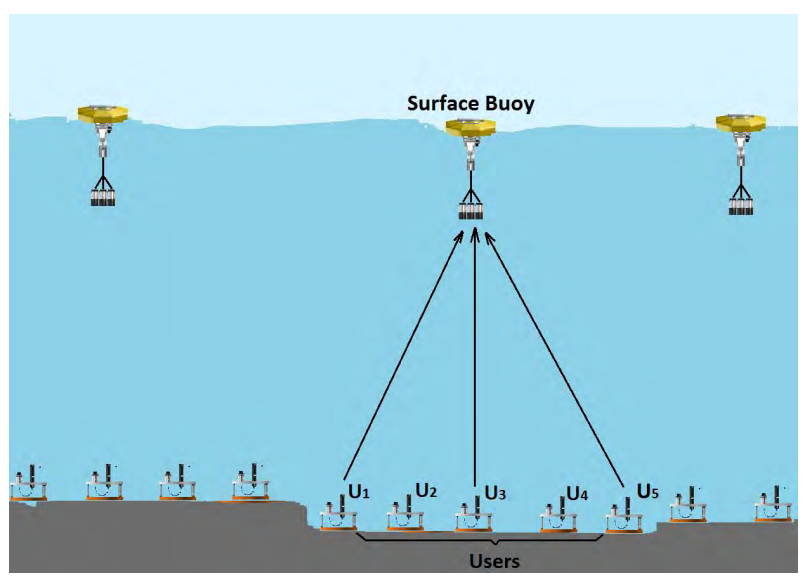

Figure 1: An underwater multi-user uplink communication network, where the surface buoy works as the receiver to collect the data from the bottom nodes $u_{1}$ to $u_{5}$.

Due to the space limitation and the high costs associated with underwater instrument, we suppose that each bottom node in UANs may only has a single transducer. We also assume that the receiver has multiple hydrophones for data reception, since the size of a surface buoy is usually much larger than an underwater node. Now denote the number of hydrophones on the buoy and the amount of underwater users in the coverage of the receiver as $M_{r}$ and $L$, respectively. Let $K$ 
denote the number of decodable packets in simultaneous receptions at the receiver, where $K \leq L$.

We start from a more general case of multi-user uplink networks first, where each user can have $N$ transducers with $N \geq 1$. Denote the circularly symmetric complex Gaussian noise at hydrophone $i$ of the receiver as $\mathbf{n}_{i} \in \mathbb{C}^{M_{r} \times 1}$, where $\mathbf{n}_{i} \sim N(0, \mathbf{I})$ and let $\mathbf{H}_{i}^{\dagger} \in \mathbb{C}^{M_{r} \times N}$ be the channel matrix of user $i$. By using the successive decoding scheme [19] in the multiuser multiple input multiple output (MIMO) uplink communications, the sum-rate capacity of the channel with transmission power constraints $\mathbf{P}=\left(P_{1}, \ldots, P_{K}\right)$ is given as $[20]$

$$
\mathcal{C}_{\text {uplink }}\left(\mathbf{H}_{1}, \ldots, \mathbf{H}_{K}, \mathbf{P}\right)=\max _{\operatorname{Tr}\left(\mathbf{Q}_{i}\right) \leq P_{i}, \forall i} \log \left|\mathbf{I}+\sum_{i=1}^{K} \mathbf{H}_{i}^{\dagger} \mathbf{Q}_{i} \mathbf{H}_{i}\right|,
$$

where each of the matrices $\mathbf{Q}_{i}$ is an $N \times N$ positive semidefinite covariance matrix. After the derivation, the sum-rate of the successive decoding in (1) can be written as [21]:

$$
\mathcal{C}_{\text {uplink }}\left(\mathbf{H}_{1}, \ldots, \mathbf{H}_{K}, \mathbf{P}\right) \leq \min \left(M_{r}, K\right) \mathcal{C}_{T D M A}\left(\mathbf{H}_{1}, \ldots, \mathbf{H}_{K}, \mathbf{P}\right)
$$

where

$$
\mathcal{C}_{T D M A}\left(\mathbf{H}_{1}, \ldots, \mathbf{H}_{K}, \mathbf{P}\right) \geq \log \left(1+\sum_{i=1}^{K} P_{i}\left\|\mathbf{H}_{i}\right\|^{2}\right),
$$

is the maximum average rate that can be achieved by time division scheme between single-user transmissions with constant power $P_{i}$.

From (2) and (3) we observe that, in order to maximize the sum-rate capacity of an underwater multi-user uplink network with $N=1$, the number of packets simultaneously arrived at the receiver should be equal to the amount of hydrophones, i.e., $K=M_{r}$. Here we call $K<M_{r}$ and $K>M_{r}$ as the starving reception and the supersaturated reception respectively, both of which may degrade the throughput and the energy efficiency of a network.

\section{Motivation}

In conventional multi-user uplink MAC protocols, people usually use either the handshaking or the feedback mechanism to prevent the starving and the supersaturated receptions $[9,8]$. However, both mechanisms may work ineffectively in underwater environments due to the following reasons.

(a) Collisions among control packets: In acoustic modems, a preamble segment is embedded in each outgoing packet for the purpose of automatic-gain-control (AGC), packet detection and channel estimation. When taking into account the preamble signal, the length of control packets 
in UANs is usually more than half a second [11], three orders of magnitude longer than that in terrestrial networks. Therefore, collisions among control packets in handshaking processes become nonnegligible. Especially at a high traffic load scenario, the high collision probability among control packets may significantly degrade the network performance in terms of throughput and energy efficiency.

(b) Unavailable of instantaneous CSI: In multi-user uplink communications, instantaneous CSI (e.g., the multipath response, channel gain and receiving SNR) information is generally used for adaptive transmission to optimize the communication capacity $[22,23]$. However, in oceans the instantaneous CSI may not be available due to the long packet transmission time, large propagation delay and high dynamic of acoustic channel [24]. With these three features, there can be a large divergence between the estimated channel state at the transmitter side and the actual one when a packet arrives at the receiver.

(c) Unexpected reception: In a terrestrial multi-user uplink network, packets sent in the same slot can arrive at the receiver simultaneously in light of the ignorable propagation delays. However, in underwater networks due to the low propagation speed of the acoustic signal, packets sent by different users simultaneously may arriver at the receiver at different time, and vice versa. Thus the number of packets received by the receiver may be different from the number of active senders. An example is shown in Fig. 2. Assume the surface buoy can decode at most three overlapped packets. The distances from the surface buoy to sender $u_{1}, u_{2}, u_{4}$ and $u_{5}$ are 1500 meters, 1500 meters, 750 meters and 750 meters, respectively. The duration of each data packet is 0.5 seconds. If the senders $u_{2}$ and $u_{4}$ are arranged to send data following the transmission of $u_{1}$ and $u_{5}$, there are only two active senders at each time slot. In radio networks with negligible propagation delays, there are only two packets received by the receiver in each time slot. However, since in water sender $u_{1}$ and $u_{5}$ to the surface buoy have 0.5 seconds longer propagation delay than $u_{2}$ and $u_{4}$, all packets from the four users arrive at the receiver at the same time, causing a supersaturated reception. We call it as the the unexpected reception problem, which potentially degrades the throughput and the energy efficiency in underwater networks.

(d) Low propagation speed: The sound speed in water is only about 1,500 m/s, five orders of magnitude lower than the electromagnetic wave in air. The low acoustic speed may cause the following problems to conventional multi-user uplink MAC protocols. 


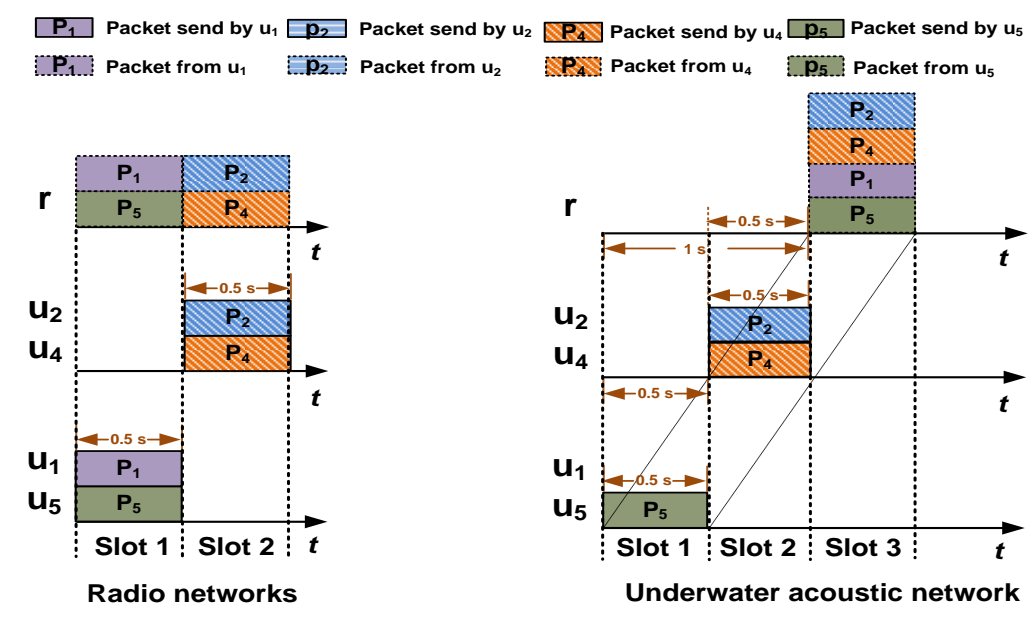

Figure 2: Unexpected reception problem in underwater multi-user uplink communication networks, where $r$ is the surface buoy and $u_{i}$ is the user $i$ in Fig. 1 .

- Low channel utilization: In a handshaking or feedback based multi-user uplink MAC, the time spend on waiting for the control packets is considerable because of the low propagation speed of acoustic signal. This greatly reduces the channel utilization of these protocols.

- Fairness issue: In an underwater multi-user uplink network, the difference of propagation delays between the receiver and different senders cannot be ignored anymore. If we use a terrestrial protocol like [8] and [9] in oceans, a user further from the receiver need to wait a longer time to get a response (handshaking or feedback message) than the closer one. In this case, the transmission opportunities of users are heterogeneous, which lead to a fairness problem.

The aforementioned issues motivate us to propose a new MAC protocol for underwater multiuser uplink networks with a fair data transmission, high channel utilization and low energy consumption.

\section{CT-MAC Protocol}

In CT-MAC, we suppose that the network is time synchronized ${ }^{1}$, which can be done by using the approach proposed in [25] at the initial stage of a network. In this section, we first introduce

\footnotetext{
${ }^{1}$ The overhead of time synchronization in UANs is significantly higher than that in radio networks. However, the collision free data transmission and the high channel initialization of CT-MAC may compensate for the additional overhead on running a time synchronization protocol.
} 
how CT-MAC works in one-dimensional uplink networks. Then we extend the protocol to the two-dimensional scenario.

\subsection{One-dimensional Uplink Networks}

A typical one-dimensional network is a string topology network, where acoustic nodes are deployed in a line, as shown in Fig. 1. This topology is widely envisioned in the oil pipeline monitoring and coastline protection systems.

In CT-MAC, the time is divided into slots with equal length. Each time slot is composed of a competition cycle and a data transmission cycle.

\subsubsection{The Competition Cycle}

In the competition cycle, each user performs the following three tasks:

(a) Generate a random value ${ }^{2}$ as a priority level (PL) to compete for a sending opportunity. If a user does not have any packet to send, it sets its PL as zero.

(b) Deliver the PLs to its direct neighbors instead of broadcasting to the whole network to reduce the energy consumption.

(c) If a user has received PLs from its neighbors in previous competition cycles, it works as a helper to relay these PLs in the current competition cycle.

In a certain competition cycle, if all users know the PLs of all others, they can decide to send the data or not independently in the following data transmission cycle by comparing the value of PLs. It is easy to have that with the multihop propagation, a PL need at most $L-1$ time slots to propagate to the whole network, where $L$ is the number of users in a string network.

In conventional MAC protocols, the competition is processed in a serial fashion, where a new round of competition will not start until the old one is complete. A typical example is the handshaking based MAC, in which a user sends a request-to-send (RTS) packet to an intended receiver and waits for the response, e.g. a clear-to-send (CTS) packet. During the waiting time, even if this user generates a new data packet to the same receiver, it will not initiate a new round of competition until the previous handshaking process is ended.

\footnotetext{
${ }^{2}$ More complicated competition schemes for fair transmissions will be introduced in Section 5 .
} 
If we use the serial competition scheme in CT-MAC, the channel utilization will be very low, since each competition needs $L-1$ time slots for PLs propagating to the whole network. Therefore, we propose a parallel competition scheme for our protocol. In this scheme, the competition is continuous. Even if the PLs produced by a user in previous time slots have not yet reached the whole network, new PLs will still be generated and sent in each competition cycle.

Let $\mathrm{CC}_{i}$ and $\mathrm{TC}_{i}$ denote the competition cycle and the data transmission cycle in the $i^{\text {th }}$ time slot, respectively. Denote $\mathrm{PL}_{i, j}$ as the PL generated by user $j$ in $\mathrm{CC}_{i}$. In Fig. 3 , we use three users as an example to show the details of PL propagation in our parallel competitions. In $\mathrm{CC}_{1}$, the PL produced by each user in the first time slot is only known by the most direct neighbors. Starting from $\mathrm{CC}_{2}$, users in the network not only generate and transmit new PLs in the current competition cycle, but also help to relay old PLs which they received in the previous competition cycles to their neighbors. It is easy to observe that in $\mathrm{CC}_{2}$, all PLs produced in $\mathrm{CC}_{1}$ are available to all users.

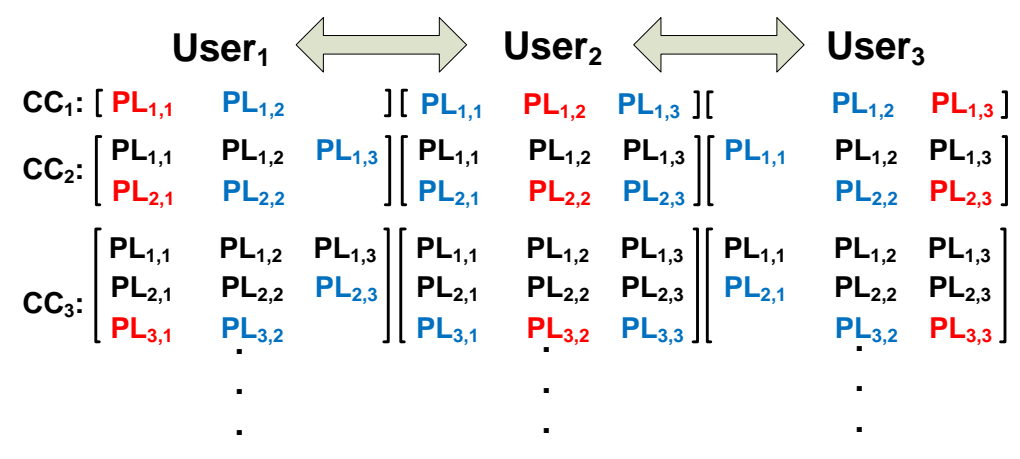

Figure 3: The PL transmission scheme in CT-MAC, where the red, blue and black colors represent the newly generated PLs, the overheard PLs from direct neighbors and the historical PLs, respectively.

In general, if there are $L$ users in one-dimensional uplink networks, PLs generated at $\mathrm{CC}_{i}$ can be received by all users at $\mathrm{CC}_{i+L-2}$. This implies that in the very beginning $L-2$ time slots of CT-MAC, no data can be transmitted without a common view on PL competitions. We call these $L-2$ time slots as the delaying slots.

Since users in CT-MAC need to help relaying the PLs from others, a user may push out multiple PLs in one competition cycle. Here we assume in $\mathrm{CC}_{i}$, user $j$ embeds all PLs it needs to send in the current competition cycle into the competition packet $(\mathrm{CP}), \mathrm{CP}_{i, j}$. In order to avoid collisions among CPs, each user does a random backoff before transmitting the CP. 


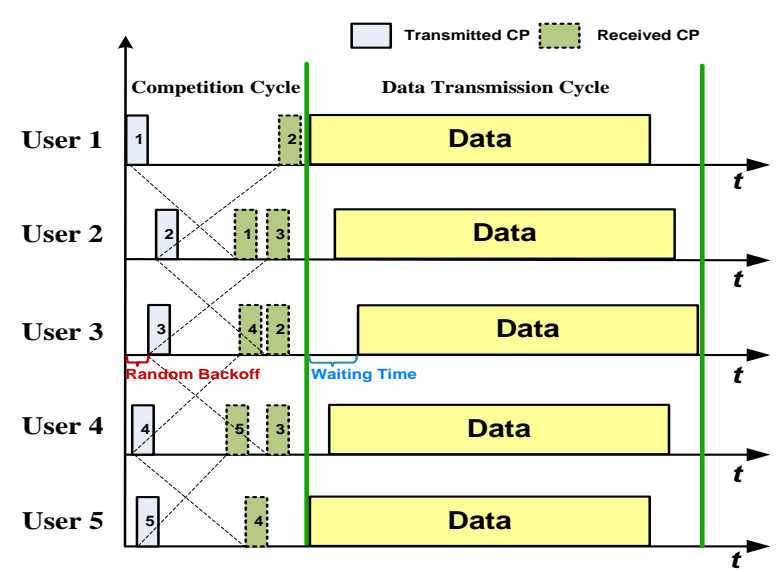

(a) TS 1

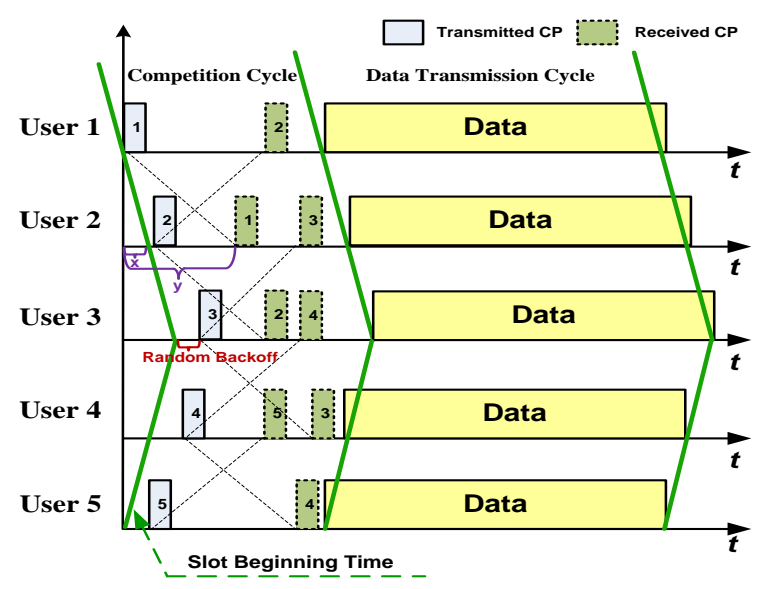

(b) TS 2

Figure 4: Comparison between two transmission schemes

\subsubsection{The Transmission Cycle}

To avoid the supersaturated reception, only $K$ out of $L$ users with the highest $\mathrm{PL}_{i-L+2, j}$ are allowed to send their data in $\mathrm{TC}_{i}$, where $i=L-1, L, L+1, \ldots$ and $j=1,2, \ldots, L$.

Here we want to make packets from different users arrive at the receiver simultaneously ${ }^{3}$. In this way, the unexpected reception described in Section 3 can be avoided. To achieve this goal, we design two transmission schemes for CT-MAC. Following denote:

- $c$ as the sound speed in water,

- $d_{i}$ as the distance between the receiver and sender $i$,

- $t_{c c}$ as the length of a competition cycle,

- $t_{t c}$ as the length of a data transmission cycle,

- $T_{s}$ as the length of a time slot (sum of $t_{c c}$ and $t_{t c}$ ),

- $t_{c p}$ as the transmission time of a CP,

- $t_{b o}$ as the maximum value of the random backoff,

- $t_{d p}$ as the transmission time of a data packet,

- $l_{i, j}$ as the distance between sender $i$ and sender $j$,

- $l_{\max }$ as the maximum distance between any two neighbors,

- $\Delta d_{\max }$ as the maximum difference between distances from the receiver to any two neighbors.

\footnotetext{
${ }^{3}$ If packets from different users arrive at the receiver not exactly at the same time, the packets are still decodable, but with degraded decoding performance. The study on the misalignment symbol timing on OFDM based multiuser uplink communications can be found in [5] and [7].
} 
$d_{i}, l_{i, j}$ can be measured at the initial stage of the network by using a classic two-way handshaking method, which is generally used in sender-receiver based time synchronization protocols [25]. Similar distance measurement approach has been successfully tested in the sea experiment [26]. $l_{\max }$ and $\Delta d_{\max }$ are available at the receiver based on the measurements of $d_{i}$ and $l_{i, j}$, respectively.

- Transmission Scheme 1 ( $\left.\mathrm{TS}_{1}\right)$ : All users in $\mathrm{TS}_{1}$ start their time slot simultaneously. To make packet from different senders reach the receiver at the same time, a user with a shorter $d_{i}$ to the receiver need to wait for the one with longer $d_{i}$ before the data transmission, as shown in Fig. 4(a). Using five users in Fig. 1 as an example, the distance between $u_{3}$ and the receiver is the shortest among the five users. The waiting time of $u_{3}$ before its data transmission should be $\max \left\{d_{i}-d_{j}\right\} / c$, where $i \neq j$ and $i, j=1,2, \ldots, L$. Hence, the minimum $t_{t c}$ and $t_{c c}$ are $\max \left\{d_{i}-d_{j}\right\} / c+t_{d p}$ and $l_{\max } / c+t_{c p}+t_{b o}$, receptively. Finally, we have

$$
T_{s 1}=t_{c c}+t_{t c}=\frac{\max \left\{d_{i}-d_{j}\right\}+l_{\max }}{c}+t_{d p}+t_{c p}+t_{b o} .
$$

- Transmission Scheme $2\left(\mathrm{TS}_{2}\right)$ : Different from $\mathrm{TS}_{1}$, we allow users in $\mathrm{TS}_{2}$ to start their time slots at different time for more efficient data transmissions. As shown in Fig. 4(b), a user with a larger $d_{i}$ starts its time slot ahead of a user with a smaller $d_{i}$. For user $i$, the advanced time of its time slot, which is denoted as $x_{i}$, is $x_{i}=\left(d_{i}-\min \left\{d_{j}\right\}\right) / c$, where $j=1,2, \ldots, L$. With this scheme, we have $t_{t c}=t_{d p}$. The minimal $t_{c c}$ should be $t_{c c}=\left(\Delta d_{\max }+l_{\max }\right) / c+t_{c p}+t_{b o}$ to guarantee that the competition cycle is long enough for all CPs reception. Finally, we have

$$
T_{s 2}=t_{c c}+t_{t c}=\frac{\left(\Delta d_{\max }+l_{\max }\right)}{c}+t_{d p}+t_{c p}+t_{b o} .
$$

In $\mathrm{TS}_{2}$, since users do not start their time slot at the same time, the competition cycle of a user may overlap with part of the data transmission cycle of its neighbors. Therefore we should ensure that there are no sending-receiving collisions between data packets and CPs. Using $u_{1}$ and $u_{2}$ in Fig. 4(b) as an example, if a CP from $u_{1}$ arrives at $u_{2}$ earlier than the slot beginning time of $u_{2}$, i.e., $y<x$, the reception of this CP may conflict with $u_{2}$ 's data transmission. Next, we prove that this collision does not happen in CT-MAC.

Proof: $y \times c=l_{1,2}$, and $x \times c=d_{1}-d_{2}$. Let users $u_{1}, u_{2}$ and the receiver be the three vertexes of a triangle, then $l_{1,2}, d_{1}$ and $d_{2}$ are three sides of this triangle. According to the triangle inequality principle that the sum of the lengths of any two sides of a triangle always exceeds the length of the third side, we have $l_{1,2}+d_{2}>d_{1}$. Hence, we have $l_{1,2}>d_{1}-d_{2}$, i.e., $y>x$. 
The length of a time slot in $\mathrm{TS}_{2}$ is reduced by $\max \left\{d_{i}-d_{j}\right\}-\Delta d_{\max }$, which improves the channel utilization of the protocol. Finally, the channel utilization of CT-MAC with $\mathrm{TS}_{2}$, which is denoted as $U$, is

$$
U=\frac{K t_{t c}}{T_{s}}=\frac{K c t_{d p}}{\Delta d_{\max }+l_{\max }+c\left(t_{d p}+t_{c p}+t_{b o}\right)} .
$$

It is worth noting that in the conventional single-input single-output (SISO) network, the channel utilization is less than one. However, in multi-user network, this value may be larger than one, since the network supports multiple users to send data simultaneously.

\subsection{Two-dimensional Uplink Networks}

CT-MAC can be easily extended to two-dimensional uplink networks. Its competition mechanism in a two-dimensional network is the same as in one-dimensional networks. By using the parallel competition scheme, $K$ out of $L$ users with the highest PLs are allowed to send their data in each data transmission cycle. However, in order to transmit CPs effectively while avoiding the supersaturated reception in a two-dimensional network, the transmission scheme $\mathrm{TS}_{2}$, which is originally designed for one-dimensional networks, need a slight modification.

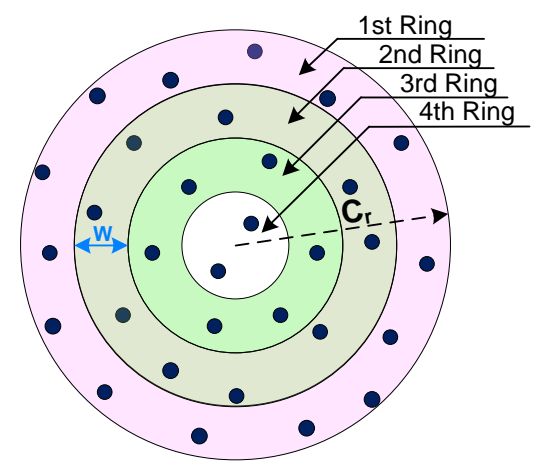

Figure 5: A general two-dimensional uplink network, where the coverage of the receiver is a cycle with the radius $C_{r}$.

In a two-dimensional network, we divide the coverage area of the receiver into several concentric rings, as shown in Fig. 5. The width of each rings is equal, which is denoted as $W$. Now, we set $W=d_{\text {avg }}$, where $d_{\text {avg }}$ is the average distance between neighboring users in the two-dimensional network. Let $r_{i}$ and $d_{i}^{\prime}$ denote the $i^{\text {th }}$ ring and the average distance from the receiver to users in the $i^{\text {th }}$ ring, respectively.

Since the distances between the receiver and users in the same ring are close, all users in the same ring start their time slot at the same time. Moreover, users in an outer ring start their 
time slots earlier than the ones in an inner ring with an advanced time depending on the distance difference to the receiver. Referring to $\mathrm{TS}_{2}$ in Section 4.1.2, the advanced time of users in the ring $r_{i}$, which is denoted as $x_{i}^{\prime}$, is $x_{i}^{\prime}=\left(d_{i}^{\prime}-\min \left\{d_{i}^{\prime}\right\}\right) / c$.

Similar to $\mathrm{TS}_{2}$ in an one-dimensional networks, a random backoff before a CP transmission is also required in the two-dimensional network to avoid collisions among CPs. Therefore, in each competition cycle, a user can successfully receive CPs from its neighbors not only in the same ring but also in neighboring rings. If there are no isolated users, PL produced by each user can propagate to the whole network within several delaying slots, the number of which is less than or equal to $L-2$.

Let $\Delta d_{\text {max }}^{\prime}$ denote the maximal distance difference between the receiver and any two users in different neighboring rings, which can be calculated by the receiver at the initial stage of the network with a two-way handshaking approach. Referring to (5) we have that the minimal length of the time slot in a two-dimensional uplink network as

$$
T_{s}=\frac{\left(d_{\max }^{\prime}+l_{\max }\right)}{c}+t_{d p}+t_{c p}+t_{b o} .
$$

Replacing parameter $\Delta d_{\max }$ in (6) with $\Delta d_{\text {max }}^{\prime}$, we can get the channel utilization of CT-MAC in the two-dimensional uplink networks.

\section{Competition Schemes for Fair Transmissions}

To make the flow of CT-MAC easy to understand, in the previous section, a user just compete for sending opportunities with a random PL. However, the network may have the fairness issue with this simple strategy. In this section, we propose two specific competition schemes for CT-MAC, aiming to improve both the short-term and the long-term fairness of the network.

\subsection{Homogeneous Traffic Generation}

We start from networks with homogeneous traffic generation rates, which is a common scenario. In these networks, the number of packets generated by different users are almost the same in a long time period. In a short time, however, differences may still exist due to the randomness of the traffic generation at each user.

Here, we have two goals for the fair transmission: (a) In a network, the more packets a user accumulated in its sending queue, the higher the transmission opportunity it has to achieve the 
short-term fairness, (b) The total number of packets sent by users are close in a long period to achieve the long-term fairness. In the following two sections we introduce how to design a competition scheme to achieve these two goals.

\subsubsection{Short-term Fairness}

If each user use a random value as the PL to compete for the sending opportunity in the data transmission cycle as what we did in Section 4, transmissions in a short time may be unfair, since the PL of each user is independent with the number of packets it accumulated. Therefore, we need to adjust the basic competition scheme to improve its short-term fairness in a homogeneous traffic generation scenario.

We assume that each user generates its data packets at the beginning of a time slot. Let $g_{i, j}$ and $g_{i, j}^{\prime}$ denote the total number of packets accumulated by user $j$ at the end of $i^{\text {th }}$ time slot and the number of new packets generated by user $j$ in the $i^{\text {th }}$ time slot, respectively. Here, $g_{i, j}^{\prime}$ is supposed to follow the Poisson distribution with the mean value $\lambda_{j}$. For a homogeneous traffic generation rates network, we have $\lambda_{j} \equiv \lambda$, for $j=1,2, \ldots, L$. Therefore, the number of packets accumulated by user $j$ in the $i^{\text {th }}$ time slot, which is denoted as $A_{i, j}$, is $A_{i, j}=g_{i-1, j}+g_{i, j}^{\prime}$. Now, we define two events:

- Successful Competition Event: If user $j$ wins the competition for transmission in the $i^{\text {th }}$ time slot, we call it as a successful competition, which is denoted as $H_{i, j}^{1}$.

- Failed Competition Event: If user $j$ loses the competition for transmission the $i^{\text {th }}$ time slot, we call it as a failed competition, which is denoted as $H_{i, j}^{0}$.

In order to achieve the short-term fairness, the goal of a competition scheme is to make the conditional transmission probability of $H_{i, j}^{1}$ meet

$$
\mathrm{P}\left(H_{i, m}^{1} \mid A_{i, m}=x_{1}\right)>\mathrm{P}\left(H_{i, n}^{1} \mid A_{i, n}=x_{2}\right) \text {, if } x_{1}>x_{2} .
$$

To make (8) hold, in each competition cycle of CT-MAC, user $j$ includes not only the PL, but also the number of accumulated packets, i.e., $A_{i, j}$, into $\mathrm{CP}_{i, j}$. In the data transmission cycle, $K$ out of $L$ users with the largest $A_{i, j}$ are allowed to send their data. If several users have the same number of accumulated packets, then these users compare the corresponding PLs as described in Section 4 to determine the final data transmission. We denote this competition scheme as CS.1. 


\subsubsection{Long-term Fairness}

For a network with a homogeneous traffic generation rates, the long-term fairness can be measured by the Jain's fairness index $F_{J}$, where [27]

$$
F_{J}=\frac{\left(\sum_{j=1}^{L} \gamma_{j}\right)^{2}}{L \sum_{j=1}^{L} \gamma_{j}^{2}},
$$

where $\gamma_{j}$ is the channel occupancy rate of user $j$. The transmission of a network is fair if $F_{J}$ is close to one; otherwise, it lacks long-term fairness. In Section 9.1, we will show that CT-MAC has a good long-term fairness with the competition scheme CS.1 in a homogeneous traffic generation rates network.

\subsection{Heterogeneous Traffic Generation}

In a network with heterogeneous traffic generation rates, we assume that the traffic generation rate of user $j$, namely $g_{i, j}^{\prime}$, follows the Poisson distribution, but $\lambda_{i} \neq \lambda_{j}$, if $i \neq j$.

According to (8), if we still use CS.1 in CT-MAC, a user with a large $\lambda_{j}$ may always have larger $A_{i, j}$ in each time slot than a user with a small $\lambda_{j}$. In this case, users with small $\lambda_{j}$ may lose all competitions and have no chance to send their data, which will be shown in Section 9.1. Therefore, CS.1 is no longer appropriate for the fair transmission in a heterogeneous traffic generation rates network. For this reason, a new competition scheme is required.

\subsubsection{Short-term Fairness}

To achieve the short-term fairness in a heterogeneous traffic generation rates network, the goal of a competition scheme is to make the conditional transmission probability of $H_{i, j}^{1}$ meet

$$
\mathrm{P}\left(H_{i, j}^{1} \mid A_{i, j}=x_{1}\right)>\mathrm{P}\left(H_{k, j}^{1} \mid A_{k, j}=x_{2}\right) \text {, if } x_{1}>x_{2} .
$$

Different from (8), which is the comparison of accumulated packets for different users in the same time slot, (10) is the comparison of accumulated packets for the same user in different time slots. Users with less increment on accumulated packets (smaller $\lambda$ ) have lower transmission opportunities. In this situation, all users will have a chance to transmit. 


\subsubsection{Long-term Fairness}

To achieve the long-term fairness in a network with heterogeneous traffic generation, we require that the average channel occupancy rate of each user is proportional to its traffic generate rate ${ }^{4}$, i.e.,

$$
E\left(\gamma_{j}\right)=\frac{K \lambda_{j}}{\sum_{j=1}^{L} \lambda_{j}}
$$

From (11) we observe that the total channel occupancy rate $\sum_{j=1}^{L} E\left(\gamma_{j}\right)$ equals to $K$, which is the maximum reception capability of the receiver. If we design a competition scheme based on (11), all users will have a chance to send their data. Now, we start from $E\left(\gamma_{j}\right)$, where

$$
\begin{aligned}
E\left(\gamma_{j}\right) & =\lim _{m \rightarrow \infty} \frac{1}{m} \sum_{i=1}^{m} \mathrm{P}\left(H_{i, j}^{1}\right) \\
& =\lim _{m \rightarrow \infty} \frac{1}{m} \sum_{i=1}^{m} \sum_{x=0}^{\infty} \mathrm{P}\left(H_{i, j}^{1} \mid A_{i, j}=x\right) \mathrm{P}\left(A_{i, j}=x\right) .
\end{aligned}
$$

Several kinds of functions can be chosen for $\mathrm{P}\left(H_{i, j}^{1}\right)$ to make (10) hold, e.g. exponential function, step function and liner function. Here we use the step function as an example to design our competition scheme. We make

$$
\mathrm{P}\left(H_{i, j}^{1} \mid A_{i, j}=x\right)= \begin{cases}1, & x>u_{i, j} \\ \alpha_{i, j}, & x=u_{i, j} \\ 0, & \text { otherwise }\end{cases}
$$

where $\alpha_{i, j}$ and $u_{i, j}$ can be calculated by substituting (13) into (12), i.e.,

$$
\mathrm{P}\left(A_{i, j}>u_{i, j}\right)+\alpha_{i, j} \mathrm{P}\left(A_{i, j}=u_{i, j}\right)=\frac{K \lambda_{j}}{\sum_{j=1}^{L} \lambda_{j}} .
$$

In the competition cycle, each user includes both its $\mathrm{PL}$ and the value of $\mathrm{P}\left(H_{i, j}^{1} \mid A_{i, j}\right)$ into its CP. In the data transmission cycle, $K$ out of $L$ users with the largest $\mathrm{P}\left(H_{i, j}^{1} \mid A_{i, j}\right)$ are allowed to send the data. If there are multiple users have the same $\mathrm{P}\left(H_{i, j}^{1} \mid A_{i, j}\right)$, then these users compare their PLs for the final decision. We denote this competition scheme as CS.2. The long-term fairness of CS.2 will be evaluated in Section 9.1.

The competition scheme CS.2 requires users in each time slot to calculate their conditional transmission probabilities $\mathrm{P}\left(H_{i, j}^{1} \mid A_{i, j}\right)$ based on the number of accumulated packets. Next, we

\footnotetext{
${ }^{4}$ Although here we make $E\left(\gamma_{j}\right) \propto \lambda_{j}$, CT-MAC can support arbitrary channel occupancy pattern by modifying $K \lambda_{j} / \sum_{j=1}^{L} \lambda_{j}$ in (11) and (14) to $K v_{j}$, where $\sum_{j=1}^{L} v_{j}=1 . v_{j}$ is the target channel occupancy rate of user $j$.
} 
introduce an iterative algorithm to compute this parameter.

\section{Algorithm 1}

Initialization: Let $A_{1, j}=g_{1, j}^{\prime}$. According to the Poisson distribution of $g_{1, j}^{\prime}$ with mean value $\lambda_{j}$, we have

$$
\mathrm{P}\left(A_{1, j}=x\right)=\frac{\left(\lambda_{j}\right)^{x} e^{-\lambda_{j}}}{x !} .
$$

Substitute (15) into (13) and (14) to calculate $\mathrm{P}\left(H_{1, j}^{1} \mid A_{1, j}\right)$.

Step 1: $\mathrm{P}\left(g_{i, j}\right)$ can be computed through

$$
\begin{aligned}
\mathrm{P}\left(g_{i, j}=x\right) & =\mathrm{P}\left(H_{i, j}^{1} \mid A_{1, j}=x+1\right) \mathrm{P}\left(A_{i, j}=x+1\right) \\
& +\left[1-\mathrm{P}\left(H_{1, j}^{1} \mid A_{i, j}=x\right)\right] \mathrm{P}\left(A_{i, j}=x\right) .
\end{aligned}
$$

Step 2: By using $A_{i+1, j}=g_{i, j}+g_{i+1, j}^{\prime}$, where $g_{i, j}$ and $g_{i+1, j}^{\prime}$ are independent, we can calculate $\mathrm{P}\left(A_{i+1, j}\right)$ through

$$
\mathrm{P}\left(A_{i+1, j}\right)=\mathrm{P}\left(g_{i, j}\right) * \mathrm{P}\left(g_{i+1, j}^{\prime}\right),
$$

where $*$ is the convolution operator, and $g_{i+1, j}^{\prime} \sim \operatorname{Pois}\left(\lambda_{j}\right)$.

Step 3: Substitute $\mathrm{P}\left(A_{i+1, j}\right)$ into (14) to calculate $u_{i+1, j}$ and $\alpha_{i+1, j}$.

Step 4: Substitute $u_{i+1, j}$ and $\alpha_{i+1, j}$ into (13) to compute $\mathrm{P}\left(H_{i+1, j}^{1} \mid A_{i+1, j}\right)$.

By repeating Step 1 to Step 4 , users can calculate their $\mathrm{P}\left(H_{i, j}^{1} \mid A_{i, j}\right)$ in each time slot iteratively.

\section{Power Allocation}

The power allocation strategy aims to distribute available power among transmitters to maximize the channel capacity in the multi-user network. There have been intensive research in this area to optimize the transmission power in both terrestrial and underwater multi-user uplink networks. Since the power allocation is not the focus of this paper, we just give a brief introduction on power allocation in CT-MAC.

As we discussed in Section 3, the instantaneous CSI may not be available in underwater communications, due to the large propagation delay, long packet transmission time and high dynamic 
of acoustic channel. However, the statistical information of underwater channel can still be used for power allocation, since it is relative stable in a long time period [24].

In CT-MAC, we can measure the statistical information, like the average receiving SNR and the distribution of channel response of underwater channel at the initial stage of the network, and then consider it as a priori knowledge. In this way, existing algorithms, such as [23] and [28], can be employed by CT-MAC to assign the transmission power for underwater multi-user uplink communications.

It is worth noting that the aforementioned power allocation algorithms usually require the MAC layer to provide the information about which user will transmit. This information is available in CTMAC with its parallel competition scheme, since users completely know who wins the competition for the data sending in each time slot. This is one superior of CT-MAC when compared with some conventional MAC protocols [8, 29].

\section{The Bad Request Issue}

In this section, we introduce a special issue in CT-MAC, which may degrade the channel utilization of the protocol.

To improve the channel utilization, we designed a parallel competition scheme for CT-MAC.

Essentially, users in this scheme use their current statuses, namely $A_{i, j}, \mathrm{PL}$ and $\mathrm{P}\left(H_{i, j}^{1} \mid A_{i, j}\right)$ to request for the transmission opportunities in the future time slot. If their statuses are changed during the propagation of $\mathrm{CP}$, these transmission opportunities may be wasted. We call this problem as the bad request issue.

Using a string topology network with twenty users as an example, suppose the traffic generation rates of the network is homogeneous, and the competition scheme CS.1 is employed. Denote the time slot $i$ as $S_{i}$. We assume that in $S_{1}$, user $u_{1}$ has a data to send. According to CS.1, it sends $A_{1,1}=1$ to its neighbors. However, in the following seventeen slots, even if $u_{1}$ does not generate any new data, it still has $A_{i, 1}=1, i=2, \ldots, 18$ to compete for the transmission opportunities. This is because the competition results in $S_{1}$ are not known immediately but be delayed by 18 slots as described in Section 4. $u_{1}$ continually competes the sending opportunity for the only data packet it generated in $S_{1}$.

After delaying slots, at $S_{19}$, we suppose that $u_{1}$ wins the competition and sends out the only 
packet it accumulated in the queue. However, $u_{1}$ has attended all the competitions between $S_{2}$ and $S_{18}$. In this case, $u_{1}$ waste the sending opportunities if it wins one or more competitions in these slots, since it currently has no data to send.

We will evaluate the impact of the bad request issue on channel utilization of CT-MAC in Section 9.1

\section{The CP Loss Problem}

In CT-MAC, CP carries the competition information for each user, the successful transmission of CPs is thus important. Although we have used the backoff mechanism to prevent collisions among CPs, the reception of CP may still fail due to the poor channel caused by the large noise, long multipath and wide Doppler spread. In this section, we first evaluate the effect of the CP loss in one-dimensional networks, and then propose two feasible solutions to handle the lost CPs.

\subsection{The effect of CP loss}

In a string topology network, the quality of acoustic channels between different neighbors may have some differences. Therefore, we assume that the successful transmission probability (delivery ratio) of CPs also varies on different links.

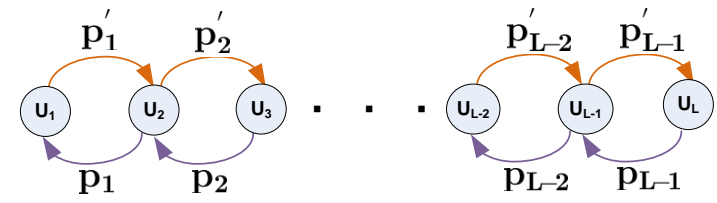

Figure 6: The delivery ratio of $\mathrm{CP}$ in a string network

Let $p_{j}$ and $p_{j}^{\prime}$ denote the delivery ratio of the CP from user $(j+1)$ to user $j$, and from user $j$ to user $(j+1)$, respectively, as shown in Fig. 6 . Denote $\mathcal{P}_{i j}$ as the delivery ratio of a $\mathrm{CP}$ from user $i$ to user $j$, where

$$
\mathcal{P}_{i j}= \begin{cases}\prod_{k=i-1}^{j} p_{k}, & i>j, \\ 1, & i=j, \\ \prod_{k=i}^{j-1} p_{k}^{\prime}, & i<j .\end{cases}
$$

If a user misses $\mathrm{CP}_{i, j}$, the corresponding competition information, such as $\mathrm{PL}_{i, j}, A_{i, j}$ and $\mathrm{P}\left(H_{i, j}^{1} \mid A_{i, j}\right)$, which is carried by this $\mathrm{CP}$, is also lost. Using $\mathrm{PL}_{i, j}$ as an example, let $w_{j}$ denote the 
number of lost PLs on user $j$ in a specific cycle. Denote the probability density of $w_{j}$ as $\mathrm{P}\left(w_{j}\right)$. It is easy to have that the probability that user $j$ successfully receive all corresponding PLs is

$$
\mathrm{P}\left(w_{j}=0\right)=\prod_{i=1}^{L} \mathcal{P}_{i j}
$$

The probability that user $j$ misses one of these PLs is

$$
\begin{aligned}
\mathrm{P}\left(w_{j}=1\right) & =\sum_{i=1}^{L}\left(1-\mathcal{P}_{i j}\right) \prod_{\substack{k=1 \\
k \neq i}}^{L} \mathcal{P}_{k j} \\
& =\sum_{i=1}^{L} \frac{1-\mathcal{P}_{i j}}{\mathcal{P}_{i j}} \prod_{k=1}^{L} \mathcal{P}_{k j} \\
& =\sum_{i=1}^{L}\left(\frac{1}{\mathcal{P}_{i j}}-1\right) \mathrm{P}\left(w_{j}=0\right) .
\end{aligned}
$$

Then the probability that user $j$ misses two of these PLs is

$$
\begin{aligned}
\mathrm{P}\left(w_{j}=2\right) & =\frac{1}{2 !} \sum_{i=1}^{L}\left(1-\mathcal{P}_{i j}\right) \sum_{\substack{k=1 \\
k \neq i}}^{L}\left(1-\mathcal{P}_{k j}\right) \prod_{\substack{m=1 \\
m \neq i, k}}^{L} \mathcal{P}_{m j} \\
& =\frac{1}{2}\left[\sum_{i=1}^{L}\left(\frac{1}{\mathcal{P}_{i j}}-1\right) \mathrm{P}\left(w_{j}=1\right)-\sum_{k=1}^{L}\left(\frac{1}{\mathcal{P}_{k j}}-1\right)^{2} \mathrm{P}\left(w_{j}=0\right)\right] .
\end{aligned}
$$

Finally, we can calculate $\mathrm{P}\left(w_{j}\right)$ iteratively with the following expression:

$$
\mathrm{P}\left(w_{j}=x\right)=\frac{1}{x} \sum_{i=1}^{x}(-1)^{i-1} \psi_{j}(i) \mathrm{P}\left(w_{j}=x-1\right),
$$

where $x=1, \ldots, L$ and

$$
\psi_{j}(i)=\sum_{k=1}^{L}\left(\frac{1}{\mathcal{P}_{k j}}-1\right)^{i} .
$$

The mean value of $w_{j}$ is

$$
E\left(w_{j}\right)=\sum_{x=1}^{L} x \mathrm{P}\left(w_{j}=x\right) .
$$

Given the analytical expression of the average number of PLs losses by each user in (24), we can evaluate the performance of CT-MAC in real network with channel loss.

If a user misses the competition information from other users, it is unable to accurately decide if it should send data or not in the following data transmission cycle. In the next section, we introduce how to handle this problem. 


\subsection{Handling the lost CPs}

In CT-MAC, we can use two simple strategies, namely, the underestimation and the overestimation, to handle the lost CPs.

In the underestimation strategy, if a user loses any other users' competition information, e.g., $\mathrm{PL}_{i, j}, \mathrm{P}\left(H_{i, j}^{1} \mid A_{i, j}\right)$ and $A_{i, j}$, it assumes that these users have the lowest priority in the competition. It is easy to understand that with the this strategy, the number of active users in each data transmission cycle is possibly larger than the maximum number of senders that a network can support, which causes a supersaturated reception.

In the overestimation strategy, a user assumes that other users, the competition information of which are lost, have the highest priority for the data transmission. With this strategy, the channel resource might be wasted. The number of active users is possibly less than the maximum number of senders that a network can support if the lost priority information is overestimated, which causes a starving reception.

Although the channel utilization is not optimal with starving reception, all packets can be successfully decoded by the receiver as long as the channel is good. By contrast, if supersaturated reception happens, the data packets will collide at the receiver, which is the worst case that we try to avoid in underwater communications. For this reason, we consider the overestimation as a wiser option than the underestimation strategy to handle the lost CPs in CT-MAC.

Now, we derive the channel utilization of CT-MAC in a homogeneous traffic rates network with the overestimation strategy. In an ideal situation, where there is no lost CP, the average transmission probability of each user in the data transmission cycle is $K / L$. When taking into account the lost CPs, this probability is reduced to $\left(K-w_{j}\right) /\left(L-w_{j}\right)$ for user $j$. Denote the average number of active users as $K^{\prime}$, we have

$$
K^{\prime}=\sum_{i=1}^{L} \sum_{x=1}^{K} \frac{K-x}{L-x} \mathcal{P}\left(w_{j}=x\right)
$$

which is less than or equal to $K$.

Finally, replacing $K$ in (6) with $K^{\prime}$, we can get the channel utilization of CT-MAC in a network with homogeneous traffic generation rates and under CP loss effect. 


\section{Simulations and Analysis}

In this section, we use the MATLAB ${ }^{5}$ as the simulation platform to evaluate the performance of CT-MAC protocol in a string network. The depth of users and the distance between neighboring nodes are 500 meters and 200 meters, respectively. In the simulation, we use two different network size, $L=10$ and $L=20$, as examples for performance analysis. The number of hydrophones on the receiver is $M_{r}=4$. We first evaluate the fairness of difference competition schemes, and show the impact of the lost CP problem and bad request issue on CT-MAC in Section. 9.1. Then, we compare the channel utilization, energy efficiency and end-to-end delay of CT-MAC with two representative multi-user MAC protocols in Section 9.2.

\subsection{Performance Evaluation}

Firstly, we evaluate the long-term fairness of competition scheme CS.1 in the network with homogeneous traffic generation rates. For comparison purposes, two different competition schemes, which are denoted as CS.3 and CS.4, are also investigated. In CS.3, $K$ out of $L$ users with the lowest $A_{i, j}$ are allowed to send their data, which is completely opposite to CS.1. In CS.4, K/2 out of $L$ users with the highest $A_{i, j}$, and another $K / 2$ out of $L$ users with the lowest $A_{i, j}$ are designed to send their data in each data transmission cycle. This competition scheme can be considered as a mixture of CS.1 and CS.3.

In Fig. 7 , we set the traffic generation rate $\lambda_{j}=1$ for $j=1,2, \ldots, 20$, in a 20 sender network and plot the Jain's fairness index $F_{J}$ for all three competition schemes. From this figure, we observe that $F_{J}$ of CS.1 is gradually close to one with the running of the protocol. This proves that CT-MAC with CS.1 achieves a good long-term fairness in a homogeneous traffic generation rate network. By contrast, $F_{J}$ of CS.3 and CS.4 are very low, close to 0.2 and 0.4 , respectively. This implies that CT-MAC may lose its long-term fairness with these two competition schemes.

In addition, we observe that the $F_{J}$ of all three competition schemes in Fig. 7 starts from 0.2. This is because no matter which competition scheme we used, after the first data transmission, if user $j$ sends the data, $\gamma_{j}$ in (9) always equal to one; otherwise, $\gamma_{j}=0$. In each data transmission

\footnotetext{
${ }^{5}$ Considering the simpleness of multi-user networks with single receiver, using MATLAB is sufficient to simulate the collision avoidance and transmission scheduling of CT-MAC and two representative multi-user MAC protocols. Therefore, we did not choose other specialized underwater network simulators, such as Aqua-Sim [30] or SUNSET [31], for performance evaluations.
} 
cycle, there are $K$ users are allowed to transmit. Therefore, $F_{J}$ of all competition schemes starts from $K / L$, which is 0.2 .

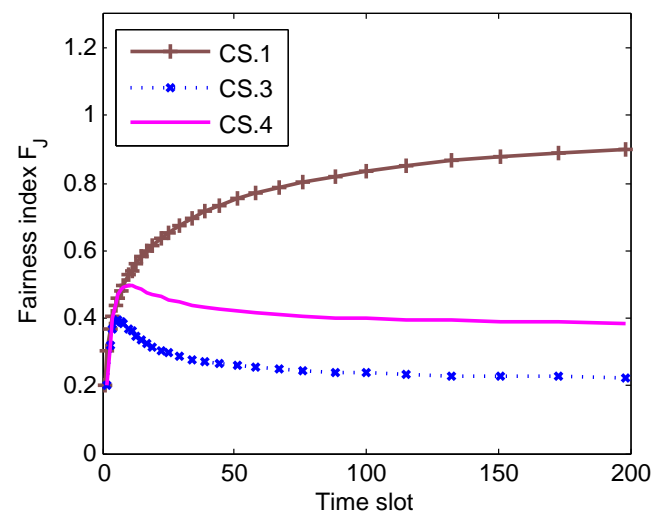

Figure 7: The long-term fairness comparison in a network with homogeneous traffic generation rates

To give us an insight into Fig. 7, we plot the distribution of the number of accumulated packets, i.e., $\mathrm{P}\left(A_{i, j}\right)$, after the protocol running 10,000 time slots, as shown in Fig. 8. If all users in the network do not send any data, then the average number of $A_{i, j}$ should equal the number of time slots, which is 10,000 , because $\lambda_{j}=1, j=1,2 \ldots, L$. In this figure, when using CS.1, we observer that $A_{i, j}$ is distributed around 8,000. More specifically, since the data transmission in CS.1 is fair in a long-term period, the packet sending opportunities of are users are almost equal, which is $K / L=0.2$. Therefore, after 10,000 time slot the average number of $A_{i, j}$ is $(1-0.2) \times 10000=8000$, which proves the long-term fairness of CS.1 again.

For comparison, when we use CS.3 in CT-MAC, a user with a larger $A_{i, j}$ is more likely to fail its next transmission competition to a user with a smaller $A_{i, j}$. This finally leads to an extremely differentiated $A_{i, j}$. Part of users, who win first, easily get the chance to send their data later on ( $A_{i, j}$ goes down to zero), and others that failed the competition at the beginning keep losing the sending opportunity $\left(A_{i, j}\right.$ goes up to 10,000). The fairness problem also occurs in CS.4, but less severe than in CS.3. This is because all users in CS.4 have a chance to send their data in each time slot, although the sending opportunities are not equal for users. More specifically, a user with a larger $A_{i, j}$ in CS.4 has less transmission opportunity than a user with a smaller $A_{i, j}$, but this opportunity is not zero. That is why the left peak of $\mathrm{P}\left(A_{i, j}\right)$ in CS.4 of Fig. 8 is 9,000 rather than 10,000, and the area of the right part in CS.4 is smaller than in CS.3.

In addition, to test the accuracy of Algorithm 1, we also compare the theoretical analysis of 

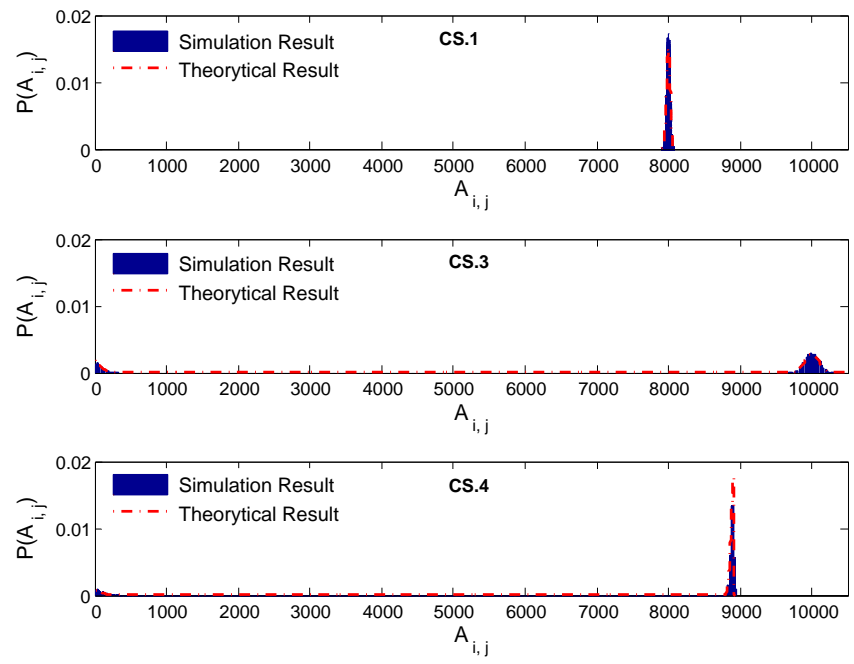

Figure 8: The distribution of $A_{i, j}$ in CT-MAC with different competition schemes

$\mathrm{P}\left(A_{i, j}\right)$ with the simulation results. From the comparisons in Fig. 8 we observer that the theoretical computations of $\mathrm{P}\left(A_{i, j}\right)$ match the simulation results very well in all three competition schemes.

In a network with heterogeneous traffics, we make $\lambda_{j}$ of different users uniformly distribute between 0.1 and 2 to evaluate the long-term fairness of CS.2. As shown in Fig. 9, the channel occupation rate of $\lambda_{j}$ conforms with (11), which is proportional to the traffic generation rates. By contrast, if we still use CS.1 in the heterogeneous traffic generation rates network, the transmission opportunities of users with small $\lambda_{j}$ may be "plundered" by users with larger $\lambda$. This can be found in Fig. 9, where the channel occupancies of the first eleven users $\left(\lambda_{j}\right.$ between 0.1 and 1$)$ are almost zero.

In Fig. 10, we test the average number of $\mathrm{CP}$ losses, i.e., $E\left(w_{j}\right)$, in a 10 sender network with varying $\mathrm{CP}$ delivery ratio, i.e., $p_{j}$ and $p_{j}^{\prime}$. From this figure we observe that $p_{j}$ affects $E\left(w_{j}\right)$ significantly. When $p_{j}=0.99$, node $u_{1}$ and $u_{1} 0$ loses $0.5 \mathrm{PLs}$ in average, which is only $5 \%$ out of the total. However, when $p_{j}$ reduces to 0.9 , the average number of lost PLs at $u_{1}$ and $u_{10}$ grows to 3.5, six times higher than the situation with $p_{j}=0.99$. Moreover, the position of a user in the network also affects its $E\left(w_{j}\right)$. Compared with users on the edge of the network, the central users have much smaller $E\left(w_{j}\right)$ owing to the shorter CP forwarding pathes. In addition. Fig. 10 also shows the consistence between the simulation results of $\mathrm{CP}$ losses and the theoretical analysis derived in (22) to $(24)$.

Fig. 11 shows the impact of lost CP on CT-MAC, where the overestimation strategy is employed. 


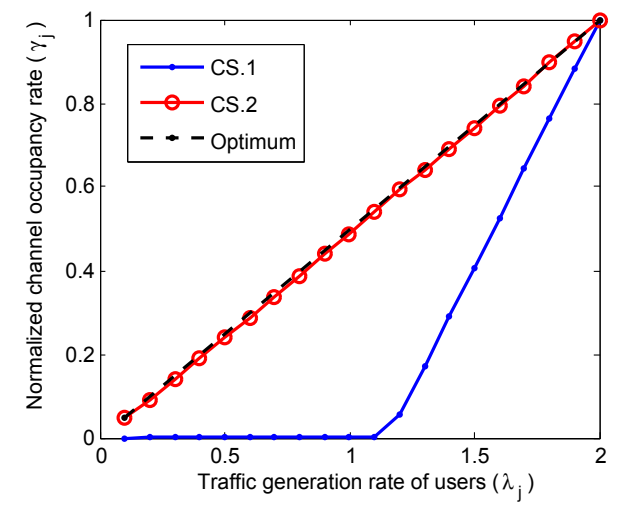

Figure 9: The long-term fairness

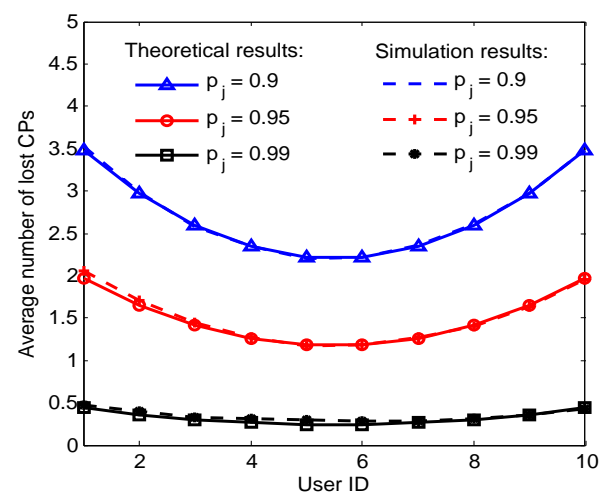

Figure 10: The average number of lost PLs

We observe an improvement on the normalized channel utilization with the reduced network size and the increased delivery ratio of CPs. This figure reveals that the multi-user uplink network with a larger number of users is much more sensitive to the lost CP than a network with smaller size. When reducing $p$ from 1 to 0.9 , the throughput of the network with 10 users degrades by $60 \%$, compared with $99 \%$ decrease for the network of 20 size. Therefore, a larger network size increases the requirement on the reliability of $\mathrm{CP}$ transmission in CT-MAC. However, it is worth noting that CP delivery ratio with 0.9 is a really low one considering the small packet size and short communication distance. According to the experiment results we collected from Long Island Sound in 2014, the CP delivery ratio is expected to be around 0.98. In this situation, the throughput degradation in the networks with 10 and 15 senders is acceptable, which is verified in Fig. 13(a).

Again, the validity of the theoretical analysis on the channel utilization of CT-MAC under the effect of CP loss is confirmed by comparing with simulation results. As shown in Fig. 11, the theoretical results derived from (6) and (25) match the simulation outcomes very well.

In Fig. 12, we evaluate the effect of bad request issue on CT-MAC. In the ideal situation, we make users in the simulation get aware of all $A_{i, j}$ information immediately. To compare with this ideal case, we plot the channel utilization of CT-MAC in the real scenario, where $L-2$ delaying slots exist in the protocol. From Fig. 12 we observe that the bad request problem slightly degrades the channel utilization of the protocol, especially in a large size network, where the PL information need more time slots to propagate to the whole network. This is because in larger networks with longer PL delivery delays, the number of users' accumulated packet is more likely to change. If users competed more transmission opportunities than they need, the bad request incurs and degrades 


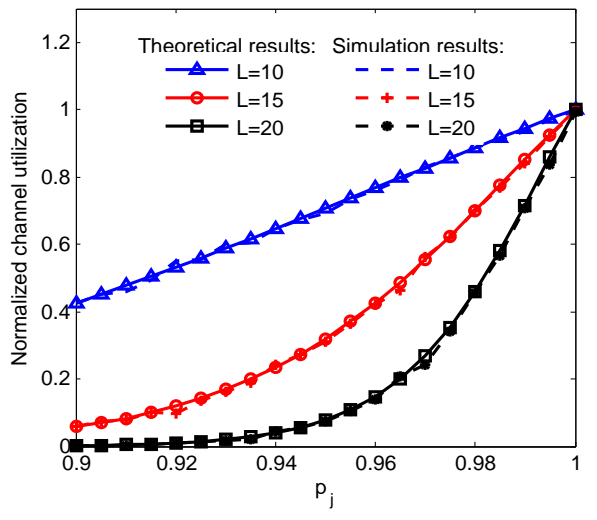

Figure 11: Channel utilization with respect to $p_{j}$

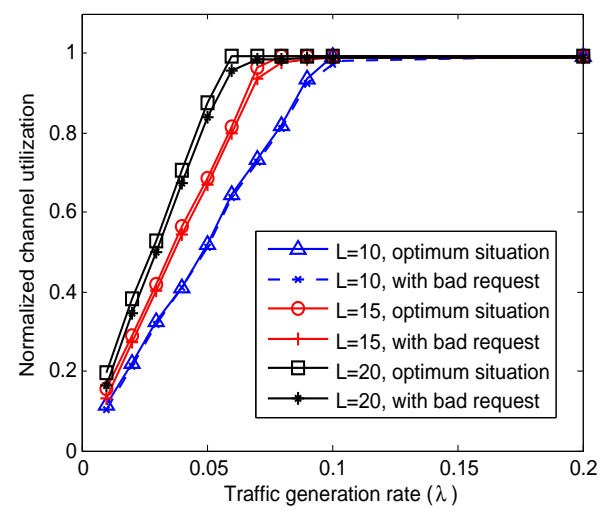

Figure 12: Effect of the bad request

the network throughput. In addition, with the increment of traffic generation rates, the effect of the bad request on the channel utilization becomes negligible. This is because in a high traffics network, the amount of accumulated packets on each user is large, whereas reduces the probability to waste transmission opportunities.

\subsection{Performance Comparison}

To verify the advantages of CT-MAC in multi-user uplink networks, we compare the channel utilization, energy efficiency and end-to-end delay of CT-MAC with two representative multi-user MAC protocols, namely, channel aware Aloha [8] and multi-antenna reception MAC [9], both of which were briefly introduced in Section. 1. Here, the channel utilization is defined as the ratio of the channel resource utilized for successful data transmission ${ }^{6}$, which can be a value larger than one in multi-user uplink networks. The energy efficiency is measured as the average power consumption on both control and data packets per each successful data delivery. The end-to-end delay measures the average delay for a data packet to be delivered to the common receiver.

The default network size we used in the following simulations is 10 . The duration of preamble signal in each packet is 0.5 seconds, which is the same as the real acoustic modem [11]. The lengthes of data and CP are 400 bytes and 40 bytes, respectively. The transmission rate of acoustic modems is $3 \mathrm{kbps}$. We use Rayleigh fading ${ }^{7}$ to model the acoustic channel with mean value $\beta_{j}$ [34], where $\beta_{j}$

\footnotetext{
${ }^{6}$ The network throughput can be represented as the channel utilization times modem transmission rate.

${ }^{7}$ There have been extensive studies on underwater horizontal channel modeling [32, 33, 34]. However, the models of underwater vertical channel are still rare. The impact of different channel models on performance of the protocols will be discussed at the end of this section.
} 
is proportional to the square of communication range, $d_{j}^{2}$. The default CP successful transmission rate is set as $p_{j}=p_{j}^{\prime} \triangleq 0.98^{8}$ for $j=1,2, \ldots, L-1$. The effect of $p_{j}$ on the performance of CT-MAC is also evaluated. Since the receiver can support a maximum of 4 simultaneous transmissions, the upper-bound of the channel utilization is $400 \%$.

From Fig. 13(a) we observe that, the channel utilizations of all three MAC protocols are very close in the low traffic scenarios $\left(0 \leq \lambda_{j} \leq 0.02\right)$. This is because with a small $\lambda_{j}$, users in all protocols have enough time to send out the generated packets. The channel utilization in this case is constrained by the traffic generation rate. However, with the incremental traffic rate, CTMAC demonstrates remarkably higher channel utilization than two representative MAC protocols, because the parallel competition mechanism of CT-MAC can avoid starving and supersaturated receptions with very low latency. By contrast, the poor collision avoidance ability of channel aware Aloha and the high latency of the handshaking in multi-antenna reception MAC lead to low channel utilization performance. We suggest that CT-MAC outperforms the random access based channel aware Aloha and handshaking based multi-antenna reception MAC in terms of channel utilization.

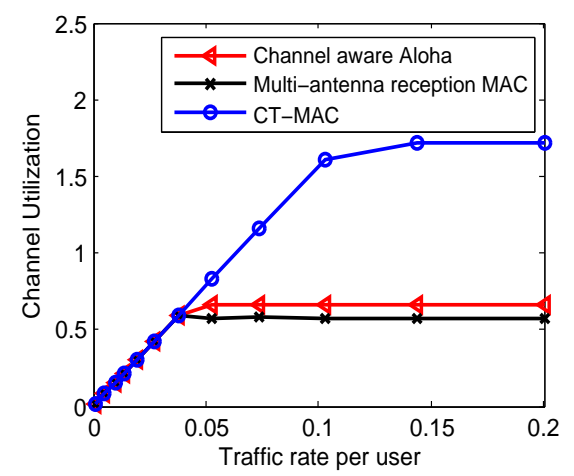

(a) Channel utilization

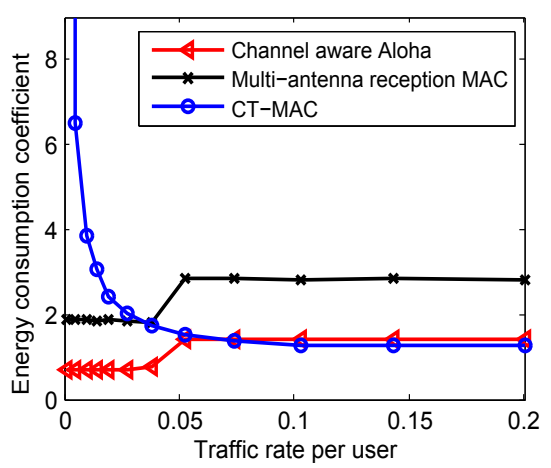

(b) Energy efficiency

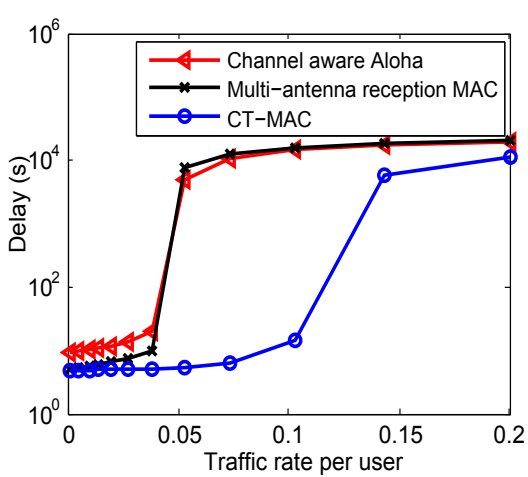

(c) Delay

Figure 13: Performance comparisons among the three protocols with $p_{j}=0.98$

In Fig. 13(b), we use the energy consumption coefficient to evaluate the energy efficiency of the protocols. The energy consumption coefficient is calculated as the energy consumption on

\footnotetext{
${ }^{8}$ According to the long term experimental results we collected at the Long Island Sound, the average delivery ratio of short packets can achieve at least $95 \%$ for the middle range (556 meters) shallow water OFDM communications [35]. Higher delivery ratio can be expected for CP transmissions due to the shorter distance (200 meters) and deeper water communication environments.
} 
single data transmission dividing the total energy consumed on each successful transmission, which takes the collision, data transmission and control packet overhead into account. A high energy consumption coefficient stands for a low energy efficiency for a protocol.

As revealed in Fig. 13(b), the energy efficiency of CT-MAC is considerably lower than the other two protocols if the traffic rate is lower than 0.038 packet per second. In the low traffic rate situation, even if a node has no data to send, it still needs to send and forward PL information in each time slot. Therefore, the large overhead on CP transmissions results in a high energy consumption coefficient for CT-MAC. However, with the growth of traffic rate, CT-MAC achieves comparable or even better energy efficiency than channel aware Aloha. The channel aware Aloha has a low energy consumption coefficient because it only allows users with the best channels to send their data, which significantly improves its energy efficiency. However, the fairness issue will be a problem, as the users with worse channels (e.g., the users at the edge of a network) always have less chance to transmit than other users. The multi-antenna reception MAC has the highest energy consumption among the three protocols due to the heavy overhead on broadcasting and retransmitting the control packets through long distance links.

Fig. 13(c) illustrates the end-to-end delays of the three protocols. When the traffic rate is lower than 0.038 packets per seconds, the end-to-end delays of both CT-MAC and muti-antenna reception MAC are around 4.9 seconds, whereas channel aware Aloha has 9.5 seconds by contrast. In CT-MAC and muti-antenna reception MAC, the end-to-end delay at low traffic loads mainly consists of the control packet and data transmissions, both of which includes the propagation delays. Channel aware Aloha has extra delays waiting for the channel going good, since only users with the good channels are allowed to transmit. This explains the higher delay for channel aware Aloha at low traffic loads in this figure. When the traffic load goes heavy, the end-to-end delay of all protocols shapely increase as the channel starts to saturate. However, for different MAC protocols, the saturated traffic rates are different. Benefitting from the high channel utilization, CT-MAC can afford a much higher traffic rate than the other two protocols without overwhelming the acoustic channel, which leads to the lowest end-to-end delay.

It is worth noting that the channel model we used in Fig. 13 is Rayleigh fading channel. This model is appropriate for the channels between the surface buoy and its furthest users, which incur almost horizontal transmissions. For users underneath the buoy, the vertical channel has much 
less multipath, and the channel models, such as Rice [32] and Gaussian fading [34], may be more appropriate than the Rayleigh model. When the channel model used in simulations does not fit the channel in the real communications, the performance of channel aware Aloha may have slight difference from what we illustrated in Fig. 13, since the transmissions are optimized based on the Rayleigh model. By contrast, using different channel models will not affect the performance of CT-MAC and muti-antenna reception MAC, since the design of these two protocols is independent with channel model. Therefore, regardless the channel model used in simulations, CT-MAC still have the highest channel utilization, lowest energy consumption and shortest delay at relatively high traffic load among the three protocols.

Table 1: Performance of CT-MAC with respect to the CP delivery ratio, $p_{j}$

\begin{tabular}{|c|c|c|c|c|c|c|c|c|}
\hline \multirow{2}{*}{} & \multicolumn{4}{|c|}{$N=10$} & \multicolumn{5}{c|}{$N=20$} \\
\cline { 2 - 9 } & \multicolumn{3}{|c|}{ Max channel utilization } & \multicolumn{2}{|c|}{ Min energy consumption } & \multicolumn{2}{c|}{ Max channel utilization } & \multicolumn{2}{c|}{ Min energy consumption } \\
\hline$p_{j}$ & Value & Percentage & Value & Percentage & Value & Percentage & Value & Percentage \\
\hline 1 & 1.94 & 100 & 7.02 & 100 & 1.84 & 100 & 6.84 & 100 \\
\hline 0.98 & 1.73 & 89.18 & 7.18 & 102.24 & 1.15 & 62.19 & 7.35 & 107.42 \\
\hline 0.95 & 1.39 & 71.37 & 7.55 & 107.49 & 0.18 & 9.53 & 14.33 & 209.40 \\
\hline 0.93 & 1.15 & 59.15 & 7.89 & 112.27 & 0.08 & 4.24 & 23.80 & 347.94 \\
\hline 0.9 & 0.83 & 42.67 & 8.73 & 124.24 & 0.04 & 2.33 & 40.09 & 585.98 \\
\hline
\end{tabular}

Fig. 11 has illustrated the significant effect of CP loss on the performance of CT-MAC. In order to give a insight into this problem, we compare the maximum channel utilization and the minimum energy consumption coefficient of CT-MAC with respect to different $\mathrm{CP}$ delivery ratios, and list the results in Table1. The percentage values are relative to the situation with no CP loss $\left(p_{j}=1\right)$. On one hand, the channel utilization of CT-MAC is remarkably affected by $p_{j}$. When $p_{j}$ decreases from 1 to 0.95 for $N=10$, the channel utilization reduces by about $30 \%$. It is acceptable as the performance of CT-MAC in this case is still better than the channel aware Aloha and multi-antenna reception MAC. However, when $p_{j}$ continuously decreases to 0.9 for $N=10$, the degradation on channel utilization will be too high, due to the significant CP losses. On another hand, the energy efficiency is less affected by $p_{j}$. Compared with the scenario with no CP loss, only $20 \%$ additional energy is consumed for $p_{j}=0.9$. This is because the CP loss will not cause the data collisions with overestimation scheme. From Table 1 we also observe that CT-MAC cannot work well in a large size network with low CP delivery ratio. For $N=20$, CT-MAC cannot work effectively when $p_{j}$ is lower 0.98 . when $p_{j}=0.9$, a majority of PLs are lost and no data can be scheduled, resulting in a 
channel utilization reduce by $97 \%$ and energy consumption increase by $486 \%$.

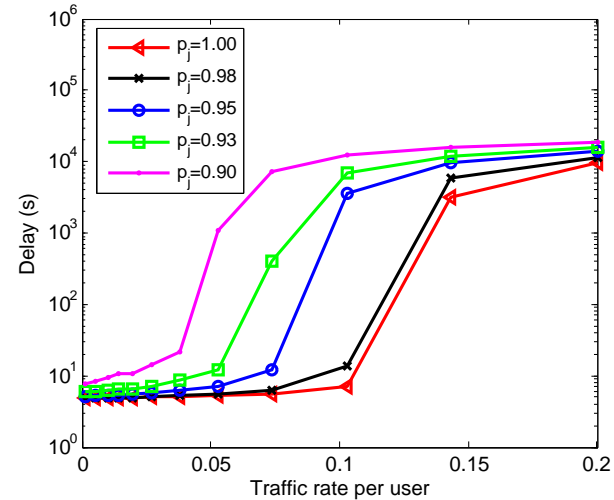

(a) $\mathrm{N}=10$

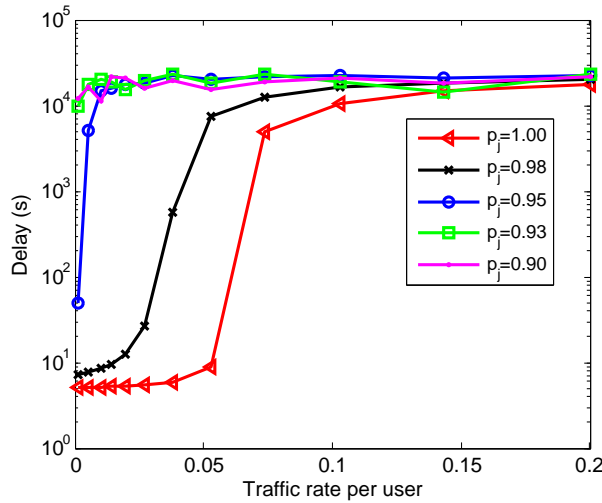

(b) $\mathrm{N}=20$

Figure 14: Delay of CT-MAC with respect to CP delivery ratio and traffic generation rate

In Fig. 14, we show the end-to-end delays of CT-MAC with respect to $\mathrm{CP}$ delivery ratio and traffic generation rate. We notice the delays have sharp increases when the traffic load reaches a certain threshold. In another word, when the traffic generation rate exceeds the network throughput, amount of packets will be accumulated and cause considerable end-to-end delays. The increase of CP loss reducing the channel utilization of CT-MAC results in lower thresholds, as displayed in Fig. 14. For example, when $N=10$ and $p_{j}=1$, the delay significantly increases around 0.12 traffic rate, compared to 0.038 with $p_{j}=0.9$. In addition, with $\mathrm{CP}$ losses, a larger network incurs incredibly longer delays at low traffic rates. It can be observed by comparing the traffic rate thresholds in Fig. 14(a) with that of in Fig. 14(b). This is because a larger network has more PL losses in average than smaller networks, causing considerable waste on data transmission opportunities in overestimation scheme. The degraded channel utilization of CT-MAC makes the packet can not be sent out timely and leads to long end-to-end delays.

The results in Table 1 and Fig. 14 reveal the fact that the reliable transmission of CP is crucially important in CT-MAC, especially in a large size network application. In order to guarantee the efficiency of CT-MAC, viable solutions could be (a) to increase the reliability of CP delivery, especially in a large network, and (b) to divide the large network to small ones, with each individually runs parallel competitions. 


\section{Conclusions}

In this paper, we have presented a competitive transmission MAC (CT-MAC) for underwater multi-user uplink communication networks. In CT-MAC, the unique features of underwater acoustic systems, such as the long propagation delay, the low transmission rate and the long preamble of acoustic modem are considered carefully. To improve the channel utilization, the energy efficiency and end-to-end delay, users in CT-MAC use a parallel competition scheme to compete for the data transmission opportunities. With this scheme, data generated by users in different time slots can join the transmission competitions in parallel, and the control packets of each user only need to reach its direct neighbors instead of the whole network.

Additionally, two competition schemes are proposed for the fair transmission in networks with both homogeneous and heterogeneous traffic generation rates. Moreover, two specific problems, namely, the bad request issue and the lost competition packet $(\mathrm{CP})$ problem in CT-MAC are studied in this paper. Solutions to address these problems are also proposed in this paper.

Finally, we evaluate the performance of CT-MAC through theoretical analysis and simulations. Compared to the channel aware Aloha and the multi-antenna reception MAC, CT-MAC has higher channel utilization under a wide range of traffic generation rates. Especially in high traffic scenarios, the channel utilization of CT-MAC is about 3.15 and 3.4 times higher than the channel aware Aloha and the multi-antenna reception MAC, respectively. Moreover, the energy consumption per each successful data transmission of CT-MAC at the high traffic rates also outperforms the other two protocols. Finally, CT-MAC has remarkably lower end-to-end delay than the channel aware Aloha and the multi-antenna reception MAC, as the high channel utilization allows CT-MAC to send packets in a much faster way without overwhelming the acoustic channel. However, the performance of CT-MAC is considerably relay on the reliable transmission of the CPs. Once the CP delivery ratio has significant reduce, the performance of CT-MAC will degrade remarkably, which should be avoided in the real applications.

\section{Acknowledgments}

This work is supported in part by the US National Science Foundation under Grant No.1018422, Grant No.1127084, Grant No.1128581, Grant No.1205665, Grant No.1208499, and Grant No.1331851. 


\section{References}

[1] I. F. Akyildiz, D. Pompili, T. Melodia, Underwater acoustic sensor networks: research challenges, Ad hoc networks 3 (3) (2005) 257-279.

[2] J.-H. Cui, J. Kong, M. Gerla, S. Zhou, The challenges of building mobile underwater wireless networks for aquatic applications, IEEE Network 20 (3) (2006) 12-18.

[3] Y. Luo, L. Pu, M. Zuba, Z. Peng, J.-H. Cui, Challenges and opportunities of underwater cognitive acoustic networks, IEEE Transactions on Emerging Topics in Computing 2 (2) (2014) 198-211.

[4] M. Stojanovic, On the relationship between capacity and distance in an underwater acoustic communication channel, ACM SIGMOBILE Mobile Computing and Communications Review 11 (4) (2007) 34-43.

[5] M. Park, K. Ko, H. Yoo, D. Hong, Performance analysis of OFDMA uplink systems with symbol timing misalignment, IEEE Communications Letters 7 (8) (2003) 376-378.

[6] A. L. Hui, K. Letaief, Successive interference cancellation for multiuser asynchronous DS/CDMA detectors in multipath fading links, IEEE Transactions on Communications 46 (3) (1998) 384-391.

[7] Z. Wang, S. Zhou, J. Catipovic, P. Willett, Asynchronous multiuser reception for OFDM in underwater acoustic communications, in: OCEANS, IEEE, 2012, pp. 1-6.

[8] X. Qin, R. Berry, Exploiting multiuser diversity for medium access control in wireless networks, in: INFOCOM, IEEE, 2003.

[9] N. Sato, T. Fujii, A MAC protocol for multi-packet ad-hoc wireless network utilizing multi-antenna, in: CCNC, IEEE, 2009, pp. 1-5.

[10] J. Partan, J. Kurose, B. N. Levine, A survey of practical issues in underwater networks, ACM SIGMOBILE Mobile Computing and Communications Review 11 (2007) 23-33.

[11] L. Pu, Y. Luo, Y. Zhu, Z. Peng, J.-H. Cui, S. Khare, L. Wang, B. Liu, Impact of real modem characteristics on practical underwater MAC design, in: OCEANS, IEEE, 2012, pp. 1-6.

[12] A. Song, M. Badiey, H. Song, W. S. Hodgkiss, M. B. Porter, et al., Impact of ocean variability on coherent underwater acoustic communications during the Kauai experiment (KauaiEx), The Journal of the Acoustical Society of America 123 (2008) 856.

[13] L.-C. Kuo, T. Melodia, Distributed medium access control strategies for MIMO underwater acoustic networking, IEEE Transactions on Wireless Communications 10 (8) (2011) 2523-2533.

[14] T. Yoo, A. Goldsmith, Capacity and power allocation for fading MIMO channels with channel estimation error, IEEE Transactions on Information Theory 52 (5) (2006) 2203-2214.

[15] D. P. Palomar, J. M. Cioffi, M. A. Lagunas, Uniform power allocation in MIMO channels: a game-theoretic approach, IEEE Transactions on Information Theory 49 (7) (2003) 1707-1727.

[16] D. Gesbert, M. Shafi, D.-s. Shiu, P. J. Smith, A. Naguib, From theory to practice: an overview of MIMO space-time coded wireless systems, IEEE Journal on Selected Areas in Communications (2003) 281-302.

[17] N. Mohamed, I. Jawhar, J. Al-Jaroodi, L. Zhang, Sensor network architectures for monitoring underwater pipelines, Sensors 11 (11).

[18] B. Li, S. Zhou, M. Stojanovic, L. Freitag, P. Willett, Multicarrier communication over underwater acoustic 
channels with nonuniform Doppler shifts, IEEE Journal of Oceanic Engineering 33 (2) (2008) 198-209.

[19] T. M. Cover, J. A. Thomas, Elements of information theory, John Wiley \& Sons, 2012.

[20] A. Goldsmith, S. A. Jafar, N. Jindal, S. Vishwanath, Capacity limits of MIMO channels, IEEE Journal on Selected Areas in Communications 21 (5) (2003) 684-702.

[21] N. Jindal, A. Goldsmith, Dirty-paper coding versus TDMA for MIMO broadcast channels, IEEE Transactions on Information Theory 51 (5) (2005) 1783-1794.

[22] W. Yu, W. Rhee, J. M. Cioffi, Optimal power control in multiple access fading channels with multiple antennas, in: ICC, 2001.

[23] A. Soysal, S. Ulukus, Optimum power allocation for single-user MIMO and multi-user MIMO-MAC with partial CSI, IEEE Journal on Selected Areas in Communications 25 (7) (2007) 1402-1412.

[24] L. Pu, Y. Luo, H. Mo, J.-H. Cui, Z. Jiang, Comparing underwater mac protocols in real sea experiment, in: Networking, IFIP, 2013.

[25] A. A. Syed, J. S. Heidemann, Time Synchronization for High Latency Acoustic Networks, in: INFOCOM, 2006.

[26] S. N. Le, Y. Zhu, J.-H. Cui, Z. Jiang, Pipelined Transmission MAC for String Underwater Acoustic Networks, in: WUWNet, ACM, 2013.

[27] R. Jain, The art of computer systems performance analysis, Vol. 182, John Wiley \& Sons Chichester, 1991.

[28] W. Rhee, J. M. Cioffi, Ergodic capacity of multi-antenna Gaussian multiple-access channels, in: Conference Record of the Thirty-Fifth Asilomar Conference on Signals, Systems and Computers, IEEE, 2001.

[29] L. X. Cai, H. Shan, W. Zhuang, X. Shen, J. W. Mark, Z. Wang, A distributed multi-user mimo mac protocol for wireless local area networks, in: GLOBECOM, IEEE, 2008, pp. 1-5.

[30] P. Xie, Z. Zhou, Z. Peng, H. Yan, T. Hu, J.-H. Cui, Z. Shi, Y. Fei, S. Zhou, Aqua-Sim: an NS-2 based simulator for underwater sensor networks, in: OCEANS, IEEE, 2009, pp. 1-7.

[31] C. Petrioli, R. Petroccia, SUNSET: Simulation, emulation and real-life testing of underwater wireless sensor networks, IEEE, 2012, pp. 12-14.

[32] F. Ruiz-Vega, M. C. Clemente, P. Otero, J. F. Paris, Ricean shadowed statistical characterization of shallow water acoustic channels for wireless communications, arXiv preprint arXiv:1112.4410.

[33] W.-B. Yang, T. Yang, Characterization and modeling of underwater acoustic communications channels for frequency-shift-keying signals, in: OCEANS, IEEE, 2006, pp. 1-6.

[34] M. Chitre, J. Potter, O. S. Heng, Underwater acoustic channel characterisation for medium-range shallow water communications, in: OCEANS, Vol. 1, IEEE, 2004, pp. 40-45.

[35] Y. Luo, L. Pu, Z. Peng, Z. Shi, J.-H. Cui, Exploring RSS Based Secret Key Generation in Underwater Acoustic Networks with Sea Experiments, Tech. Rep. Technical Report: UbiNet-TR14-01, UCONN CSE (2014). 